\title{
The real-time measurement and control system of the motor used on electric vehicle based on LabVIEW
}

\author{
Zhiyu Huang ${ }^{a}$, Yaxue Yan ${ }^{b}$ and Xi Peng ${ }^{c}$ \\ Chongqing University of Posts and Telecommunications, Chongqing 400065, China \\ ahuangzy@cqupt.edu.cn, byanyx324@163.com, cpengxixi124@126.com
}

Keywords: electric vehicle driving system; road situation-simulate; the test of electric vehicle driving system efficiency.

\begin{abstract}
The hardware platform of real-time measurement and control system composes of PXI, WT3000, the dynamometer, the tested motor, the controller and so on. Its PC platform's software is compiled by LabVIEW. The remote control, dynamic and static load output, real-time data acquisition, road situation-simulate and the test of electric vehicle driving system efficiency are its main functions. When the system is developed, its functions are verified. The results show that the system not only can satisfy the development needs of the electric vehicle driving system, but also provide great convenience for testing and improvement, because of its friendly user interface and modularized programming idea.
\end{abstract}

\section{Introduction}

As the oil crisis and environment pollution is increasingly serious, electric cars, because of its many advantages, become one of the important ways to solve the above problem [1]. At present, the electric car technology is not yet mature. The drive motor and its control system, which are important parts of electric cars, restrict the development of electric vehicles, so their development has important research significance [2]. In order to speed up the development of control system, the dynamometer is introduced to establish the test platform. In the process of development, the dynamometer can give the drive motor the corresponding load. Building on the basis, it also can simulate the traffic situations, etc... On the platform, we can collect the data which is needed, and analyze them using the appropriate methods to optimize control strategy of the motor. In order to improve the degree of automation, reliability and security of the test platform, the modular products, which are bought, are used to build the test platform. LabVIEW, the graphical programming language, is used to develop the motor real-time measurement and control system based on virtual instrument technology, which can be used in real-time monitoring and analyzing of the relative parameters.

\section{The Test Bed of Electric Vehicle Drive Motor}

In order to speed up the development of electric vehicle drive motor and its control system, reduce the cost and risk of the electric car development, a measurement and control platform of electric vehicle drive system is built based on hardware in the loop. It is mainly composed of the tested motor, controller, dynamometer, the power analyzer and the remote console.

Under the control of remote control work station, the dynamometer can load to the tested motor and simulate the traffic situations, etc. Current sensors, voltage sensors, torque sensor and other auxiliary equipment are used to transmit relevant parameters to the corresponding interface card in PXI (in the remote control work station). The PXI controller sends parameters to the industrial personal computer through the Ethernet communication. At the same time, the commands of industrial personal computer are also sent to controllers via Ethernet and CAN bus. In addition, the communication between the power analyzer and the industrial personal computer is also through Ethernet. The structure diagram is shown in figure 1. 


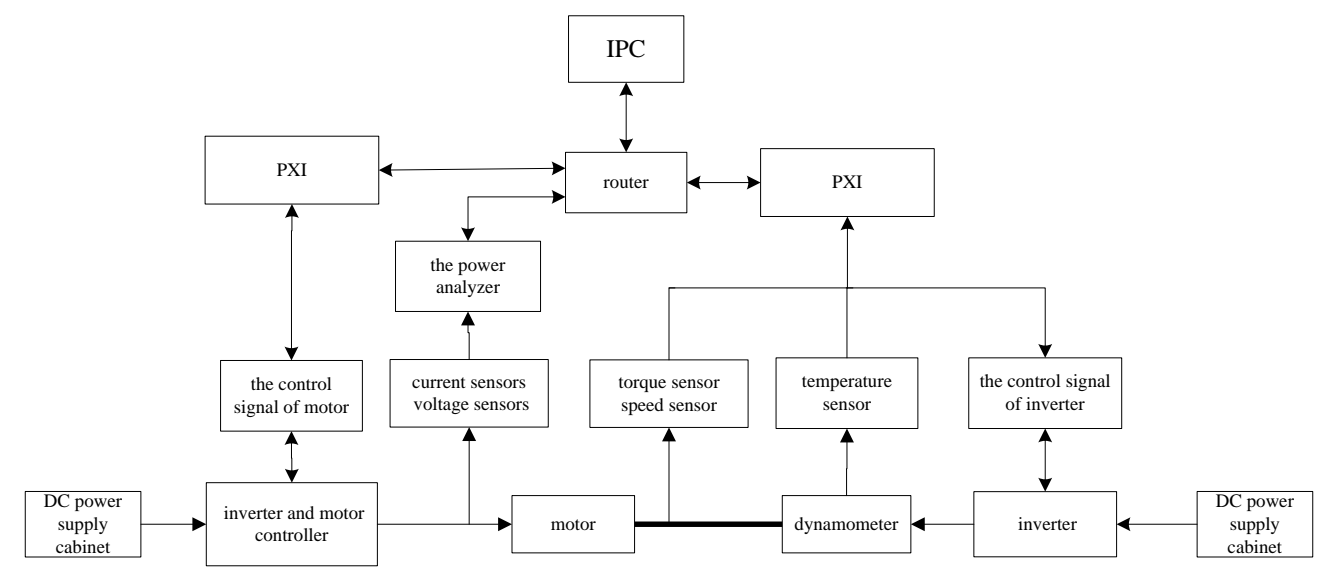

Fig. 1 The structure diagram of test platform

\section{The Functions of Measurement and Control System}

In the process of development and test, the real-time measurement and control system of motor is an important part of the motor drive system used on pure electric car. Its main functions are remote control, real-time data acquisition, dynamic load output and static load output and traffic simulation. In addition, in order to improve the efficiency of motor drive system to be advantageous for the motor control strategy optimization, the test function of the motor driven system efficiency is designed, which is introduced in detail below.

Electric vehicle drive system is the heart of the electric car. Its performance directly affects the vitality of the electric car, the rational use of limited battery energy storage and the improvement of automobile performance. So for the electric car, its design plays a key role in the whole design process [3]. To evaluate the performance of drive system, its static efficiency and dynamic efficiency should be overall considered. This is to say that the function of testing the efficiency of the motor drive system is to test its efficiency in the different working point [4]. Motor drive system mainly includes the drive motor and controller. The efficiency can be obtained by the input power of the inverter $P_{I}$ and the motor output power $P_{O}$.

$$
\eta=\frac{P_{O}}{P_{I}}=\frac{U I}{1000} / \frac{T N}{9549}=9.549 \frac{U I}{T N}
$$

Since the drive motor controller is essentially a power switch control device, which can make the power of the source convert to the suitable energy for drive motor, when the controller to choose the appropriate motor the performance of drive system depends on the drive motor if the controller is appropriate. It's means that the efficiency of the drive motor $\eta_{M}$ is obtained through the input power of the drive motor $P_{M I}$ and output power of motor shaft $P_{M O}$.

$$
\eta_{M}=\frac{P_{M O}}{P_{M I}}
$$

The drive motor on the test-bed is the three-phase AC permanent magnet synchronous motor. The motor is a three wire three-phase system, whose interior is a star connection. On the experiment platform, the wiring mode of power analyzer WT3000 is 3V3A (three-voltage, three-current), as shown in figure2.

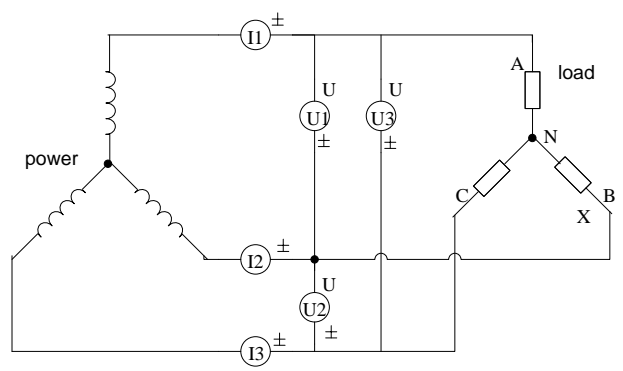

Fig. 2 The wiring mode of 3V3A 
In the figure, the wattmeter signed A, B, C is respectively connected to the three-phase load. One side of the voltage coil in wattmeter is respectively received the $\mathrm{X}$ in the graph. For derive convenient, it's supposed that any point $\mathrm{X}$ in space can be any one of the three-phase load terminals $\mathrm{A}, \mathrm{B}, \mathrm{C}$. The point $\mathrm{N}$ is the three-phase load neutral point. The average power of the three-phase load is respectively as follows:

$$
\begin{aligned}
& P_{A}=\frac{1}{T} \int_{0}^{T} u_{A N} i_{A} d t \\
& P_{B}=\frac{1}{T} \int_{0}^{T} u_{B N} i_{B} d t \\
& P_{C}=\frac{1}{T} \int_{0}^{T} u_{C N} i_{C} d t
\end{aligned}
$$

$P_{A}, P_{B} P_{C}$ - the average power of phase A, B, $\mathrm{C} u_{A N}, u_{B N} u_{C N}$-the phase voltage of phase A, B, $\mathrm{C} i_{A}, i_{B}, i_{C}$ - the line current of phase A, B, C T - the cycle of voltage signal fundamental wave.

The total power of three-phase load $P_{M I}$ :

$$
P_{M I}=P_{A}+P_{B}+P_{C}=\frac{1}{T} \int_{0}^{T}\left(u_{A N} i_{A}+u_{B N} i_{B}+u_{C N} i_{C}\right) d t
$$

In the figure, we know that:

$$
\begin{aligned}
& u_{A N}=u_{A X}-u_{N X} \\
& u_{B N}=u_{B X}-u_{N X} \\
& u_{C N}=u_{C X}-u_{N X}
\end{aligned}
$$

So,

$$
P_{M I}=\frac{1}{T} \int_{0}^{T}\left(u_{A X} i_{A}+u_{B X} i_{B}+u_{C X} i_{C}\right) d t-\frac{1}{T} \int_{0}^{T} u_{N X}\left(i_{A}+i_{B}+i_{C}\right) d t
$$

By Kirchhoff's current law, $i_{A}+i_{B}+i_{C}=0$.So,

$$
P_{M I}=\frac{1}{T} \int_{0}^{T}\left(u_{A X} i_{A}+u_{B X} i_{B}+u_{C X} i_{C}\right) d t
$$

The point $\mathrm{X}$ is any point in space. If point $\mathrm{X}$ is on the point $\mathrm{A}$,

$$
P_{M I}=\frac{1}{T} \int_{0}^{T}\left(u_{B A} i_{B}+u_{C A} i_{C}\right) d t
$$

If point $\mathrm{X}$ is on the point $\mathrm{B}$,

$$
P_{M I}=\frac{1}{T} \int_{0}^{T}\left(u_{A B} i_{A}+u_{C B} i_{C}\right) d t
$$

If point $\mathrm{X}$ is on the point $\mathrm{C}$,

$$
P_{M I}=\frac{1}{T} \int_{0}^{T}\left(u_{A C} i_{A}+u_{B C} i_{B}\right) d t
$$

In the formula $u_{B A}, u_{C A} u_{A B} u_{C B} u_{A C}$ and $u_{B C}$ are respectively the line voltage of three phase load. Above three formulas, it is known that the sum of any two wattmeter is the total power of the three-phase load. This is the principle of two watts measurement three-phase three-wire system to measure the input power.

\section{Result of the Test and Analysis}

On the basis of the test bench, the functions of the real-time measurement and control system of the motor are verified in the end. The dynamometer and power analyzer are controlled through the Ethernet communication. The data collected is shown in the figure 3, 4. 


\begin{tabular}{|l|l|l|l|l|l|l|l|l|}
\hline parameter & measured value & unit & parameter & measured value & unit & paramete: & measured value & unit \\
\hline VDC & 151.6 & V & IDC & 12.4 & A & PI & 1879.8 & "H \\
\hline VAB & 147.5 & V & VBC & 146.1 & V & VCA & 145.9 & V \\
\hline IA & 11.25 & A & IB & 11.34 & A & IC & 11.56 & A \\
\hline$\eta$ controler & 92.5 & X & $\eta$ motor & 55.9 & X & & & \\
\hline T & 20.1 & H. II & n & 499 & r/min & PMO & 1050.4 & " \\
\hline
\end{tabular}

Fig.3 the collected data

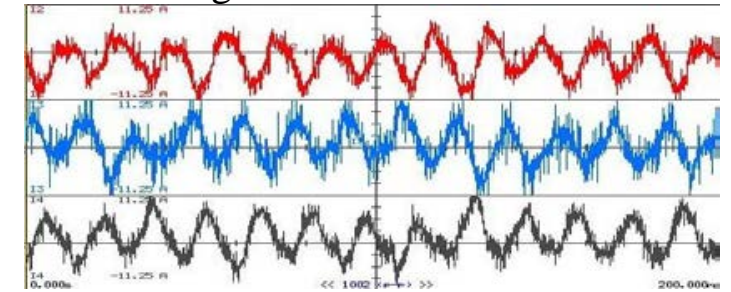

Fig.4 three phase current from power analyzer

The speed of Dynamometer is constant and the torque of motor is constant. When the speed of tested motor is different, the output torque is also changing. In this case, the work area of motor can be obtained to draw the work points of the tested motor, as shown in figure 5 . Hence the efficiency of each point is available. Using mathematical interpolation, the MAP diagram is obtained, as shown in figure 6 . The area, where controller efficiency is more than $80 \%$, to the total area ratio is an important evaluation indicator. By the MAP diagram known, the motor and its controller meet the requirements.

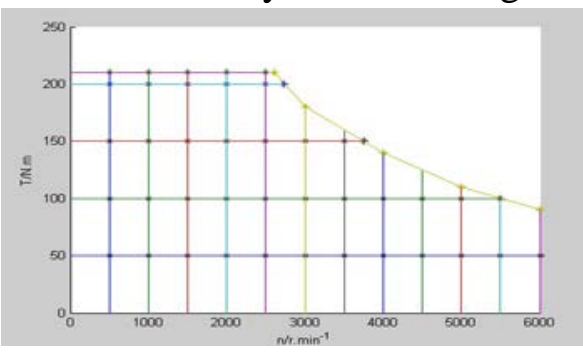

Fig.5 the test points of motor

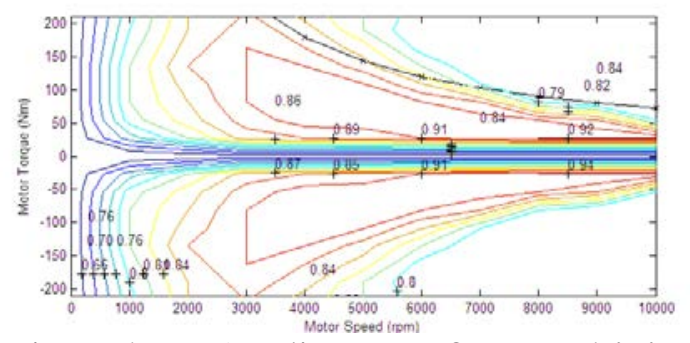

Fig. 6 the MAP diagram of motor driving system

\section{Conclusion}

The PC platform of motor drive system test bench is developed visa LabVIEW. The dynamometer and power analyzer are controlled through the Ethernet communication. At the same time, the relative data is real-time acquired. The motor is controlled through the CAN bus. Vehicle longitudinal dynamics model is established, so that the driving cycles can be simulated on the PC platform, such as the NEDC driving cycles. At last, the efficiency of the drive system was tested to draw the MAP figure, which is advantageous to the optimization of control strategy.

\section{Acknowledgement}

This research was financially supported by the key technology of pure EV motor control and drive system research and design (cstc2014yykfA4004).

\section{Reference}

[1] Chan C. The state of the art of electric and hybrid vehicles [J]. Proceedings of the IEEE, 2002, 90(2): p.247-275.

[2] M. E. Tian. The development trend of Electric vehicle [J]. Light vehicle technology, 2010 (11): p.4-6.

[3] C. P. Zhang, C. N. Zhang, Q. Song. Efficiency testing research on electromotor drive system driver working districts of electric vehicle [J]. Journal of micro motor, 2007, 40 (1): p.84-86.

[4] S. H. Sun. The design and development of the test-bed to evaluate electric car motor drive characteristic [D]. Tianjin University, 2005. 